Results: 151 patients participated: 50 with RA (90\% women, mean age $55.12 \pm$ 13.64 years), 51 with AS ( $51 \%$ women, $52.59 \pm 12.15$ years) and 50 patients with SLE (96 \% women, mean age $47.14 \pm 11.3$ years). The most frequent comorbidities were: arthritis, visual impairment, anxiety and depression (table 1). These results present a greater tendency to depression and anxiety patients of SLE. No significant differences were observed in most of the social questionnaires analyzed between groups (table 2), except in a worse mobility in patients with RA and AD compared to SLE $(p=0.017)$. About half of the patients in all groups had depression (43\%) and reduced mobility (63.6\%). All groups are satisfied with their social role $128(85.3 \%)$, have the capacity to participate in social activities $140(94 \%)$ and feel accompanied 147 (97.4\%). On the contrary, the social isolation figure is $42(28 \%)$. Social isolation implies an affectation of the serious social role in patients who claim to be accompanied, so it is not secondary to loneliness or lack of family support.

In the multivariate analysis it was observed that the independent variables that were associated with the ability to participate in social activities were satisfaction with social relations $(\beta=0.349[p=<0.001])$, mobility $(\beta=0.309[p=<0.001])$, depression $(\beta=-0.186[p=<0.011])$ and social isolation $(\beta=-0.195(p=0.001))$. This model would explain $32 \%$ of the variability in the ability to participate in social activities. $(\mathrm{R} 2=0.32)$.

Conclusion: The predictors of the ability to participate in social activities in patients with RA, AD and SLE were: depression, mobility deficit, social isolation and satisfaction with social activities. Patients with RA, AD and SLE present similar data, so there are no differences due to pathologies in the social role, highlighting that they have a good social support and despite this there is social isolation being able to be associated with the deficit in mobility and high rates of depression.

Disclosure of Interests: None declared

DOI: 10.1136/annrheumdis-2020-eular.845

\section{SAT0622-HPR \\ SAFETY IN PATIENTS WITH RHEUMATOID ARTHRITIS IN BIOLOGICAL TREATMENT OVER 65 YEARS OF AGE}

A. Crespo Golmar ${ }^{1}$, C. Moriano ${ }^{1}$, I. González Fernández ${ }^{1}$, X. E. Larco Rojas ${ }^{1}$ A. López Robles ${ }^{1}$, T. Pérez Sandoval ${ }^{1}$. Service, Rheumatology, Leon, Spain

Background: A bias has been described with the lowest prescription of biologic treatments (bDMARD) in patients with rheumatoid arthritis (RA) in the elderly, despite presenting activity rates comparable to young population and higher risk of functional disability. This could be due to concerns about co-morbidities and polypharmacy1.

Objectives: 1) To define the characteristics of patients with $R A \geq 65$ years and bDMARD to follow up in the Day Hospital of University Assistance Complex of León during the last year. 2) To record the incidence rate (IT) and ratio of incidence rates (RDI) of infections, neoplasms and cardiovascular events (CD) during the course of your therapy.

Methods: Observational, retrospective study of patients diagnosed with RA according to ACR 1987 and/or ACR 2010 criteria in intravenous biological treatment during 2019 with $\geq 65$.

Results: 40 patients with an average age at diagnosis of $55.9 \pm 15.76$ years were included, $67.5 \%$ of them were women. The average duration of the disease was $17.65 \pm 13.15$ years. $40 \%$ had a history of smoking, $35 \%$ hypertension, $20 \%$ dyslipemia and $20 \%$ diabetes mellitus. A $97.5 \%$ were positive FRRA, $57 \%$ positive ACPA, $37.5 \%$ nodular and $65 \%$ erosive. As for pre-treatment, $70 \%$ had been with conventional (cDMARD) $\geq 2$ DMARD (Methotrexate (MTX) $(92.5 \%)$ and Leflunomide $(60 \%)$ ). The mean dose of prednisone was $8.79 \pm 10.14 \mathrm{mg} /$ day. The incidence rate of infections was $1.5 \%$, and neoplasms and CD were $0.75 \%$ per person-years. The age at the beginning of the first bDMARD was $67.45 \pm 8$ years, the second $(n=20) 67.98 \pm 6.64$ and the third $(n=7) 71.79 \pm 7.49$. The first biological was a $52.5 \%$ anti-TNF, 5\% anti-CTLA4, 30\% anti-CD20 and $12.5 \%$ antilL6 (25\% monotherapy and combined with MTX 57.5\%). The second was $30 \%$ anti-TNF, $25 \%$ antiCTLA4, 15\% antilL6 and 30\% antiCD20 (50\% in monotherapy and $40 \%$ methotrexate); with the third anti-TNF $42.85 \%$, antiCTLA4 $14.29 \%$, antilL6 $14.29 \%$ and antiCD20 $28.57 \%$ (42.86\% in monotherapy and 42.46 with methotrexate). The mean doses of prednisone were $6.08 \pm 6.82,4.38 \pm 7.21$ and $6.95 \pm 5.94 \mathrm{mg} /$ day respectively. The IT of bDMARD infections were $8.81 \%, 19.81 \%$ and $7.4 \%$ person-years; of neoplasia $1.04 \%$, 0 and 0 ; and EC $3.63 \%, 0$ and 1.85 person-years. The RTIs with first, second and third biological infections were: $5.88,13.25,4.95$; with neoplasms 1.38; with EC 1.38, 0 and 0.69 . The mean total accumulated corticosteroid dose was $17.69 \pm 15.01 \mathrm{mg} /$ day

Conclusion: 1) Patients over 65 years old receiving bDMARD in our Day Hospital in 2019 were long-standing RA with aggression data, who had not responded to $\geq 2$ cDMARD and required medium-high doses of prednisone.

2) In our sample there is a link between incidence of infection and the introduction of biological therapy, which is maintained with the increasing age of our patients, and it is not so clear with neoplasms and CD. These data are consistent with the existing literature ${ }^{1,2,3}$

3) Larger, comparative studies with RA under 65 years are needed, but it is reasonable to conclude that if bDMARD is required, elderly patients could be a high-risk group for infections, requiring special monitoring and follow-up.

\section{References:}

[1] Alla Ishchenko, Rik J. Lories. Safety and Efficacy of Biological Disease-Modifying Antirheumatic Drugs in Olfer Rheumatoid Arthritis Patients: Staying the distance. Drugs Aging 2016;(33):387-398.

[2] Atsuko Murota, Yuko Kaneko, Kunihiro Yamaoka y Tsutomu Takeuchi. Safety of Biologic Agents in Elderly Patients with Rheumatoid Arthritis. J Rheumatol 2016; (43): 1984-1988.

[3] Kosuke Ebina, Motomu Hashimoto, Wataru Yamamoto, Toru Hirano, Ryota Hara, Masaki Katayama et al. Drug tolerability and reasons for discontinuation of seven biologics in elderly patients with rheumatoid arthritis. The ANSWER cohort study. PLoS ONE 14 (15):e0216624

Disclosure of Interests: None declared

DOI: 10.1136/annrheumdis-2020-eular.5260

\section{SAT0623-HPR THE LIVED EXPERIENCES OF COGNITIVE DYSFUNCTION IN ADULTS WITH FIBROMYALGIA: A QUALITATIVE SYSTEMATIC REVIEW}

S. Derham ${ }^{1}$, J. Lewis ${ }^{2,3}$, E. Dures ${ }^{4,5}$, F. Cramp ${ }^{4} .{ }^{1}$ Royal United Hospitals Bath NHS Foundation Trust, RNHRD Rheumatology Therapies, Bath, United Kingdom ; ${ }^{2}$ University of the West of England, Department of Allied Health Professions, Bristol, United Kingdom; ${ }^{3}$ Royal United Hospitals Bath NHS Foundation Trust, Complex Regional Pain Syndrome Service, Bath, United Kingdom; ${ }^{4}$ University of the West of England, Centre for Health and Clinical Research, Bristol, United Kingdom; ${ }^{5}$ Bristol Royal Infirmary, Department of Nursing and Midwifery, Bristol, United Kingdom

Background: Adults with fibromyalgia frequently report symptoms of cognitive dysfunction, often referred to as fibrofog. However primary research exploring cognitive dysfunction in the lives of adults with fibromyalgia is very limited (Kravitz and Katz, 2015).

Objectives: The aim of this review was to (i) synthesise the qualitative literature on the lived experiences of cognitive dysfunction in adults with fibromyalgia, (ii) develop common themes through thematic analysis and (iii) identify knowledge gaps to inform future research and clinical practice in this area.

Methods: Seven electronic databases (MEDLINE, Embase, CINAHL, Psy cINFO, Amed, Scopus and OpenGrey), reference lists of key articles and two high impact qualitative journals were searched from 1990 to November 2018. Articles were eligible for inclusion if they reported primary qualitative data exploring the experiences of cognitive dysfunction in adults with fibromyalgia. Included studies were appraised using the Critical Appraisal Skills Programme (CASP) qualitative checklist and extracted data analysed using narrative synthesis. SD conducted critical appraisal and data extraction on all included studies. FC, JL and ED reviewed five papers each. All papers were reviewed by two co-authors. Of the 1413 records identified, 15 studies were selected for inclusion.

Results: These studies included 208 women and 22 men with fibromyalgia, aged 18 to 72 years and representing seven different countries. Duration of diagnosis was four months to 34 years. Fourteen studies used interviews and one used focus groups. None of the included studies focussed exclusively on cognitive function in adults with fibromyalgia. Three studies identified themes specific to cognitive dysfunction and fibromyalgia symptoms. The remaining 12 studies presented relevant data intertwined with the overall lived experiences of fibromyalgia.

Cognitive dysfunction, as a part of fibromyalgia, was often unpredictable. Problems with memory and concentration that were most commonly reported were emotionally distressing and affected functional and vocational activities. Participants found communication effortful, with a negative impact on work, leisure and social activities. Stress, fear and worry around perceived cognitive changes were commonly expressed. Lost employment or changed work roles and relationships, due to cognitive difficulties, had negative impacts for many participants. The terms cognitive dysfunction and fibrofog were used interchangeably within the studies, but lacked common definition. This introduced uncertainty around whether participants and authors were describing the same phenomenon.

Conclusion: Adults with fibromyalgia experience unpredictable and emotionally impactful difficulties related to cognitive dysfunction. Functional impact was broad-reaching, particularly around work ability and lost employment opportunities. It is unclear how cognitive symptoms in fibromyalgia related to co-morbid symptoms such as pain, fatigue and poor sleep. Further research focusing on the full impact of cognitive function on the lives of adults with fibromyalgia is recommended to inform clinical practice. Research to establish clarity of definition of the terms cognitive dysfunction and fibrofog within fibromyalgia is highly recommended. References: 
[1] Kravitz H, Katz R. Fibrofog and fibromyalgia: a narrative review and implications for clinical practice. Rheumatology International. 2015;35(7):1115-25.

Acknowledgments: This work is supported by the National Institute for Heath Research [ICA-PCAF-2018-01-078 to SD]

Disclosure of Interests: Sandra Derham: None declared, Jenny Lewis: None declared, Emma Dures Grant/research support from: Independent Learning Grant from Pfizer, combined funding for a research fellow from Celgene, Abbvie and Novartis, Paid instructor for: A fee from Novartis to deliver training to nurses., Fiona Cramp: None declared

DOI: 10.1136/annrheumdis-2020-eular.2119

\section{SAT0624-HPR THE IMPACT OF PSORIATIC ARTHRITIS ON FOOT HEALTH AND INDICATION OF PODIATRY NEED IN A SECONDARY CARE SETTING}

R. Field ${ }^{1}$, S. Bannon ${ }^{2}$, K. Pouliase ${ }^{3}$, S. Mukherjee ${ }^{4} .{ }^{1}$ Dorset HealthCare University Foundation Trust, Bournemouth, United Kingdom; ${ }^{2}$ Dorset Healthcare University Foundation Trust, Podiatry, Bournemouth, United Kingdom; ${ }^{3}$ Dorset Healthcare University Foundation Trust, Bournemouth, United Kingdom; ${ }^{4}$ Royal Bournemouth \& Christchurch NHS Foundation Trust, Bournemouth, United Kingdom

Background: Psoriatic Arthritis (PsA) is a rheumatic disease affecting $0.19 \%$ of the UK population (1). It is characterised by asymmetric oligoarticular or polyarticular peripheral arthritis or axial disease with or without associated peripheral arthritis (2). Foot manifestations of synovitis, enthesitis, dactylitis and skin and nail involvement (3) are reported. Hyslop et al. have previously reported high levels of foot involvement but low current access to foot care (4). Outcome measures that include specific PsA related foot features do exist, e.g. Leeds Enthesitis Index, Tender Dactylitis Count (5). However there is currently no measure of foot involvement and impact in PsA (6).

Objectives: To identify the impact of PsA on foot health and indication of podiatry need in a secondary care outpatient setting

Methods: convenience sample was taken from a consultant rheumatologist's outpatient clinic and screened. Only those with a diagnosis of PsA were included. Sampling was conducted over a ten-week period. Screening was done using the Swindon Foot and Ankle Questionnaire (SFAQ) (7), visual Analogue Scale (VAS), clinical judgement of need for podiatric intervention and the trust's eligibility criteria for routine podiatric care.

Results: The sample $(n=16)$ was $31.3 \%$ male with a median age of 59 years (range 28-81).

Table 1. Footcare/Podiatric need identified

\begin{tabular}{lc}
\hline & Percentage (\%) \\
\hline Orthotic intervention, acute or routine care & 81.3 \\
Already being met & 25.0 \\
Eligible for care in podiatry primary care service & 81.3 \\
\hline
\end{tabular}

Table 2. SFAQ results

\begin{tabular}{lc}
\hline & $\begin{array}{c}\text { Percentage } \\
\text { Yes (\%) }\end{array}$ \\
\hline During the past week have your feet or ankles: & 68.8 \\
Been painful? & 62.5 \\
Been Swollen? & 81.3 \\
Made walking difficult? & 50.0 \\
Made standing up difficult? & 27.3 \\
Stopped you going to work? & 42.9 \\
Made other daily activities difficult? & 31.3 \\
Do your shoes rub the skin on your feet or ankles? & 50.0 \\
Do you have callus or hard, dry skin? & 25.0 \\
Have you had your footwear adapted or insoles made? & 18.8 \\
Have you had surgery, or are you waiting for surgery, on your feet or ankles? & \\
\hline
\end{tabular}

Conclusion: Of this patient group, $81.3 \%$ had a variety of foot care needs but these were being met in a limited number of cases (25\%). Far more patients $(81.3 \%)$ were eligible for care in the local trust's primary care podiatry service but were not engaging with this. $50 \%$ of the sample reported difficulty standing in the past week and $27.3 \%$ found their foot pain stopped them from going to work, indicating a clear need for foot health intervention.

Recommendations:

-Raise awareness of availability of podiatric care for PsA patients among patients and secondary care staff.

- Ensure adequate resources are allocated to manage this cohort of patients at a service provision level.

- Further research involving PsA patients referred into podiatry to assess the impact of podiatric intervention.

References:
[1] Ogdie A et al.(2012) Prevalence and treatment patterns of psoriatic arthritis in the UK. Rheumatology. 7;52(3):568-75.

[2] Cantini F, et al.(2010) Psoriatic arthritis: a systematic review. Int J Rheum Dis.13(4):300-17.

[3] Huynh D and Kavanaugh A. (2015) Psoriatic arthritis: current therapy and future approaches. Rheumatology. 54:20-8.

[4] Hyslop E et al. (2010) Foot problems in psoriatic arthritis: high burden and low care provision.Ann Rheum Dis.69(5):928

[5] Assessing psoriatic arthritis in your clinic - trainer manual. $2017 \mathrm{https}: / / \mathrm{www}$ psoriatic-arthritis.co.uk/assessmenttools.aspx (accessed 22-6-19)

[6] Carter $\mathrm{K}$ et al. (2019) Linking the patient experience of foot involvemen related to psoriatic arthritis to the international classification of functioning, disability and health. EULAR poster abstract THU0713-HPR

[7] Waller R et al. (2012) The swindon foot and ankle questionnaire: is a picture worth a thousand words?. ISRN rheumatology. 26;2012.

Disclosure of Interests: None declared

DOI: 10.1136/annrheumdis-2020-eular.5613

\section{SAT0625-HPR FATIGUE AND CONTRIBUTING FACTORS IN CHINESE} PATIENTS WITH ANKYLOSING SPONDYLITIS

W. Zhou ${ }^{1}$, J. Guo ${ }^{1}$, R. Zhao ${ }^{1}$, C. Dong ${ }^{1}$, Z. Gu ${ }^{1} .{ }^{1}$ Affiliated Hospital of Nantong University, Nantong, China

Background: Ankylosing spondylitis (AS) is a systemic chronic inflammatory disease, which most likely occurs in young men. It mainly affects sacroiliac joints, axia skeleton, thoracic cage and seriously decreasing quality of life in AS patients[1,2] In recent years, fatigue of $A S$ patients has been paid more and more attention[3] Fatigue is a complex feeling, diseased individuals describe fatigue as a sense of tiredness at rest, exhaustion with activity, lack of energy which affects daily work inertia or lack of endurance, or as loss of vitality. It has been confirmed that fatigue is not only a symptom but may also be quantified by fatigue scores and can be modified by various measures depending on the underlying cause[4]. However, there has been no study about fatigue in AS patients in China.

Objectives: This study aimed to evaluate the predictors of fatigue and the effects of fatigue on HR-QoL among patients with AS.

Methods: A total of 150 AS patients were involved in the study. A series of questionnaires included: Bath Ankylosing Spondylitis Disease Activity Index (BASDAI), Bath Ankylosing Spondylitis Metrology Index (BASMI), Bath Ankylosing Spondylitis Functional Index (BASFI), the $10 \mathrm{~cm}$ Visual Analog Scale (VAS) the Self-Rating Anxiety Scale (SAS), the Self-Rating Depression Scale (SDS) the Pittsburgh Sleep Quality Index (PSQI), the Health Assessment Questionnaire-Disability Index (HAQ-DI), the Short Form 36 Health Survey (SF-36) and the Fatigue Severity Scale(FSS). Independent samples t-test, Mann-Whitney U-test, Chi-square analysis, Pearson /Spearman correlation and binary logistic regression were used to analyze the data.

Results: The results demonstrated that $48.7 \%$ individuals with AS suffered from fatigue. Compared with AS patients without fatigue, AS patients with fatigue showed higher WHR $(P<0.05)$, increased BASDAI $(P<0.01)$ and poorer BASFI $(P<0.05)$. Meanwhile, AS patients with fatigue tended to have more severe pain $(P<0.05)$, higher degree of anxiety $(P=0.001)$, more serious functional disability $(P=0.001)$ and worse sleep quality $(P=0.001)$. Binary logistic regression indicated that WHR $(\mathrm{OR}=1.78, P<0.05)$, BASDAI (OR=1.34, $P=0.01)$, sleep disturbance $(\mathrm{OR}=2.35, P<0.05)$ were independent predictors of fatigue in AS patients. Additionally, the occurrence of fatigue significantly reduced the quality of life in AS patients both physically and psychologically.

Conclusion: These findings suggested that medical personnel should pay more attention to AS patients with fatigue and take effective measures to relieve fatigue. References:

[1] Law L, Beckman Rehnman J, Deminger A, Klingberg E, Jacobsson LTH Forsblad-d'Elia $\mathrm{H}$ (2018) Factors related to health-related quality of life in ankylosing spondylitis, overall and stratified by sex. Arthritis research \& therapy 20 (1):284. doi:10.1186/s13075-018-1784-8

[2] Hanson A, Brown MA (2017) Genetics and the Causes of Ankylosing Spondylitis. Rheumatic diseases clinics of North America 43 (3):401-414 doi:10.1016/j.rdc.2017.04.006

[3] Ulus Y, Akyol Y, Bilgici A, Kuru O (2019) Association of work instability with fatigue and emotional status in patients with ankylosing spondylitis: comparison with healthy controls. Clinical rheumatology 38 (4):1017-1024 doi:10.1007/s10067-018-4366-x

[4] Finsterer J, Mahjoub SZ (2014) Fatigue in healthy and diseased individuals. The American journal of hospice \& palliative care 31 (5):562-575. doi:10.1177/1049909113494748

Acknowledgments: Thanks to all the authors for their efforts and thanks to all members of the Department of Rheumatology of Affiliated Hospital of Nantong University for their helpfulness in the acquisition of data.

Disclosure of Interests: None declared

DOI: 10.1136/annrheumdis-2020-eular.6313 